\title{
Identifying patterns in psychiatric hospital stays with statistical methods: towards a typology of post-deinstitutionalization hospitalization trajectories
}

Philippe Golay ${ }^{\mathrm{ab}}$, Stéphane Morandia ${ }^{\mathrm{a}}$ Philippe Conus ${ }^{\mathrm{b}}$, Charles Bonsack ${ }^{\mathrm{a}}$

a. Service of Community Psychiatry, Department of Psychiatry, Lausanne University Hospital and University of Lausanne, Switzerland.

b. Service of General Psychiatry, Treatment and Early Intervention in Psychosis Program (TIPPLausanne), Lausanne University Hospital and University of Lausanne, Switzerland.

E-mail addresses: philippe.golay@chuv.ch, stephane.morandi@chuv.ch, philippe.conus@chuv.ch, Charles.bonsack@chuv.ch

Corresponding Author: Philippe Golay, Department of psychiatry, Lausanne University Hospital, Consultations de Chauderon, Place Chauderon 18, 1003 Lausanne, Switzerland. Tel: +41213147746; Fax: +41213141277; Email address: Philippe.Golay@chuv.ch 


\section{Conflict of interest with respect to the study and manuscript}

The authors have declared that there are no conflicts of interest in relation to the subject of the study.

\section{Role of the Funding Source}

This study was based on institutional funding. 


\section{ABSTRACT}

Purpose: Over the past fifty years, deinstitutionalization changed the face of psychiatry. However, outpatient treatment in the community does not always fit the needs of those who left institutions and sometimes leads to frequent re-hospitalizations, a mechanism known as the "revolving door" phenomenon. The study aim was to identify different typologies of hospitalization trajectories.

Methods: Records of 892 inpatients from the Department of Psychiatry of Lausanne University Hospital were analysed over a three-year period with discrete sequential-state analysis.

Results: Trajectories could be split between atypical users (4.9\% of patients totalling $30.6 \%$ of hospital days) and regular users. Within the atypical users group, three categories were identified: "Permanent stays" (3 patients totalling 6.3\% of hospital days), "long stays" (1.7\% patients / $8.6 \%$ hospital days) and "revolving door" stays (2.9\% patients / 15.8\% hospital days). The remaining $95.1 \%$ of the patients were classified into "unique episodes" $(70.0 \%$ patients / $24.5 \%$ hospital days) and "repeated episodes" (25.0\% patients / 44.9\% hospital days). Diagnoses of schizophrenia were overrepresented among heavy users.

Conclusions: Most patients went through a unique or low number of brief hospital admissions over the three years of the study. While the shift of previously institutionalized individuals towards high users of psychiatric hospital seems limited, this phenomenon should not be neglected since $4.9 \%$ of patients use about a third of hospital beds. Early identification of problematic profiles could allow the implementation of relapse prevention strategies and facilitate the development of alternatives to hospitalization such as assertive community treatment or housing first programs.

Key words: psychiatric hospitalization, patterns, revolving door phenomenon, re-hospitalization. 


\section{INTRODUCTION}

Deinstitutionalization and progress in psychiatric treatments changed important aspects of psychiatry over the past fifty years. In recent decades and in most western countries, both mental health services and the legal framework of involuntary psychiatric treatments were adapted in order to enhance patients' rights and autonomy. However, these modifications have not yielded all the expected effects. The de-institutionalisation movement made it possible for individuals suffering from mental disorders to leave hospital and re-enter the community [1]. This "rhetorical quest for community", described by Cohen [2] which juxtaposed "the bad exclusionary institution" and "the good, open, benevolent and accepting community", was quickly confronted with reality and unexpected challenges [3]. Outpatient treatment and support in the community did not always respond to the needs of patients leaving the institutions [4]. The frequent re-hospitalizations of seriously ill individuals with no access to suitable outpatient care became known as the "revolving door" phenomenon. In Switzerland, between 1992 and 2011, the number of psychiatric beds decreased by $44 \%$ (23\% between 2001 and 2015), but the number of psychiatric hospitalizations increased by 151\% (55\% between 2001 and 2015) [5], leading to important difficulties for hospitals currently designed for short admissions.

In this post-deinstitutionalization context, it is important to identify subgroups of patients for whom alternatives to hospitalizations should be developed, and the goal of this study was to explore contemporary hospitalization patterns based on recent statistical methods. The objective was to explore if different typologies of hospitalization trajectories could be identified and if such trajectories are related to resource use and various characteristics that may allow their early identification.

\section{MATERIAL AND METHODS}




\subsection{Procedure and participants}

All individuals hospitalized between January 1st 2010 and May 1st, 2011 in the Department of Psychiatry of Lausanne University Hospital were followed up during three years from day one of first hospitalization. Therefore, records were screened until April 302014 for the patients that were included at the end of the 16 months recruitment interval.

The catchment area of the Lausanne university hospital counted about 620,000 inhabitants at the time of the study. A total of 329 adult psychiatric beds were available on three geographical sites (53 adult beds / 100,000 inhabitants). For each patient, hospitalizations were aggregated into a string of 1095 digits with digits 0 (not in hospital) or 1 (in hospital) for every day over a three-year period. Each state sequence started with the first day at hospital and included the next three years of records. The records of 892 patients were analysed. An anonymized identification number allowed aggregation of different hospital stays for a given patient but rendered identification impossible for the authors. Local ethics committee approval and explicit consent were not required because analyses were conducted on existing routine institutional records containing anonymous data. Limited sociodemographic data such as age, gender, presence of personality disorder, presence of substance use disorder and main diagnostic at first hospitalization were available.

\subsection{Statistical analysis}

The hospitalization sequences were analysed with the TraMineR package for $\mathrm{R}$ which allowed discrete sequential-state/event analysis [6]. Sequences were analysed as states and the dissimilarity of each pair of sequences was computed using the Optimal Matching algorithm which generates edit distances that are the minimal cost, in terms of insertions, deletions and substitutions, for transforming one sequence into another [7]. A cluster analysis using Ward's method was performed on this dissimilarity matrix in order to group similar individual trajectories. 
Clusters were interpreted using visualization and statistical tools including a procedure implemented in the TraMineR package that allows extracting one or several sequences that could be considered representative of the cluster as a whole on a statistical basis [8]. Existence of several representative sequences may suggest within-cluster heterogeneity. The final number of clusters was based on interpretability. Due to of skewness, overall comparison between clusters in terms of average duration of hospitalisation was performed with a Kurskal-Wallis test. If significant, pairwise comparisons were performed with a series of Bonferroni-corrected MannWhitney U-tests. Similarly, ANOVA and Bonferroni corrected t-tests were used to compare age. Chi squared tests (or Fisher Exact's tests with exact or Monte-Carlo estimation when appropriate) were used to compare gender and diagnostic information. Box-plot for each group were used to illustrate between-clusters differences in average duration of hospitalisation.

\section{RESULTS}

In total, 892 patients were hospitalized for a total of 48,504 days. The sample mean age was 41.9 years $(S D=13.9)$ and $52.3 \%$ of the patients were female. Main diagnoses based on ICD-10 criteria at first hospitalization included depression $(n=315 ; 35.3 \%)$, schizophrenia $(n=$ $154,17.3 \%)$, anxiety and stress related disorder $(n=130 ; 14.6 \%)$, alcohol use $(n=102 ; 11.4$ $\%)$, personality disorder $(n=47 ; 5.3 \%)$, mania $(n=46 ; 5.2 \%)$, drug use $(n=46 ; 5.2 \%)$, dementia $(n=28 ; 3.1 \%$ ), behavioral syndromes associated with physiological disturbances and physical factors $(n=11 ; 1.2 \%)$ and no diagnostic information at discharge $(n=13 ; 1.5 \%)$.

We extracted an increasing number of clusters. The sequence of clustering is depicted in Figure 1. At the end, five meaningful clusters were identified. Firstly, trajectories could be split into regular (848 patients (95.1\%) totalling $69.4 \%$ of hospital days) and atypical users (44 patients (4.9\%) for $30.6 \%$ days). Regular users could be classified into "unique episode" (Cluster A; 625 (70.0\%) of patients totalling only $24.5 \%$ of hospital days) and "repeated 
episodes" (Cluster B; 223 patients (25.0\%) for $44.9 \%$ of hospital days). In Cluster A, the average number of stays was $1.43(\mathrm{SD}=0.90)$ and $73.9 \%$ of the patients had only one hospital stay. This group included the majority of patients, who had very moderate resource use and a single or a small number of close hospital stays during the three-year period (Figure 2). More precisely, the mean total duration of hospitalization over three years was 18.99 days. In Cluster $B$, the average number of stays was $3.00(S D=2.13)$. The total duration of hospitalization for the "repeated episodes (B)" group was considerably higher (97.66 days) and scattered over some further distant hospital stays.

Within the remaining atypical users group, three categories were identified including "long stays" (Cluster C; 15 patients (1.7\%) for $8.6 \%$ of hospital days), "revolving door" patients (Cluster D; 26 patients (2.9\%) \& 15.8\% of hospital days) and "permanent stays" (Cluster E; 3 patients totalling $6.3 \%$ of hospital days). In Cluster $\mathrm{C}$, the average number of stays was 3.07 $(S D=2.09)$ while it was $7.92(S D=3.82)$ in Cluster $D$. The average number of stays was 277.33 days for patients in the Long Stays (C) group and 293.58 days for the revolving door (D) group.

The groups differed with regard to total duration of hospitalization $(H=551.406, p<.001$; Figure 3). Unlike every possible group comparison (all $p<.05$ ), the difference between Clusters $C$ and $D$ was not significant $(p=1.000)$. While long stays $(C)$ and revolving door patients $(D)$ were indistinguishable based on the total duration of hospitalization their temporal dynamics were strikingly different (Figure 2). The distinctive feature of Cluster C was a few long hospital stays while the "revolving door" patients had more frequency and shorter hospital stays. Cluster B ("repeated episodes") and D ("revolving door" patients) shared some temporal dynamic features (multiple hospitalizations) although the number of hospital stays was higher in the revolving door group. The revolving door patients had higher total duration of hospitalization. The last cluster of "permanent stays" (E) consisted of only three patients who had an average of 
1022 days of hospitalization over the three years and ranged from 2-6 separate stays (Figure 2). The 6-clusters solution split the B group into two groups whose interpretation was unclear. The 7-clusters solution split the D group into two very similar groups. Therefore, no further clusters were extracted.

In order to gain a better insight into the clusters' characteristics, we performed a series of post-hoc group comparisons (Table 1). Firstly, we performed an overall test across the five groups. Secondly, if the overall test was significant, we compared the atypical users (4.9\% of patients; Clusters C, D \& E) to the remaining 95.1\% patients (clusters A \& B). Finally, if this test was significant, we compared $A$ versus $C+D+E$ and $B$ versus $C+D+E$ separately.

Age did not differ significantly across groups $\left(F_{(4,884)}=1.514, p=.196\right)$. Gender did also not differ overall (Fisher Exact's Test, $p=.313$ ). Main diagnoses differed significantly (Fisher Exact Test, $\mathrm{p}<$.001). Indeed, atypical users differed from regular users (Fisher Exact's Test, $p=$ .003). Unique episode patients differed from atypical users (Fisher's Exact Test, $p<.001$ ) but repeated episode patients did not differ from atypical patients (Fisher's Exact Test, $p=.533$ ). Examination of the contingency table (Table 1) revealed that patients with schizophrenia were more represented in the atypical user group (46.5\%) than in the unique episode group (11.6\%). Conversely, patients with anxiety and stress related disorders were underrepresented in the very heavy user group (7.0\%) compared to the unique episode group (18.1\%). However, presence of substance abuse (Fisher's Exact Test, $p=.447$ ) and personality disorder (Fisher's Exact Test, $\mathrm{p}=.905)$ did not differ overall.

\section{DISCUSSION}

Discrete sequential-state statistical analysis allowed the identification of five clinically meaningful hospitalization patterns clusters within our patient population over the study period. 
In this era of deinstitutionalization, most patients needed only a unique and short admission or a limited number of admissions. However, a very small minority of patients could be qualified as heavy hospital users, mobilizing close to a third of available beds. Since a large amount of resources is currently allocated to a small minority of patients, early identification or forecasting of problematic profiles could help develop relapse prevention strategies, facilitate the choice of the most adequate type of treatment and the development of alternatives to classic hospitalization. The question of the cost of care for people who suffer from long hospitalizations is a long-standing issue, which involves those who favored the opening of special asylums for these individuals to proponents of more open "boarding-out systems". With the emergence of neuroleptics and antidepressants during the 1960s, it was believed that this debate would cease, as chronicity of disorders would disappear. However, if medical transformations and institutional changes certainly modified the face of psychiatry over the past fifty years, challenges remain that are very reminiscent of the past. Despite important progress, deinstitutionalization per se has not resolved all the problems. While many patients have benefitted from more open and respectful treatments, and while the negative and chronifying impact of asylums is now mostly avoided, a number of patients (e.g. revolving door patients) do not seem to fit this new system and may still seek hospital support. Furthermore, very long hospitalizations, although uncommon, have not disappeared.

Schizophrenia was a distinctive feature of heavy resource use. It was found in another study that psychotic diagnosis and younger age predicted revolving door status [9]. However, in our sample, age was not associated with any of the different profiles. While one limitation of this study is the very limited sociodemographic data that accompanied the hospitalization records, the absence of diagnostic differences between atypical users and repeated episode patients was intriguing. Alcohol and drug abuse and non-compliance were found to be the most important factors in predicting the frequency of hospitalizations [10]. In another study, revolving 
door patients were also characterized as male, nonelderly, non-white, single, with low education and high unemployment, and from rural and small towns [11]. The existence of this cluster in our setting is consistent with a study that highlighted that the reorganization of the Danish mental healthcare system created a problem of "revolving door" schizophrenia patients who have been increasingly hospitalized but for shorter periods since the 1970s [12]. However, the number of revolving door patients remained very low in our data and concerned less than one of 10 hospitalized individuals suffering from schizophrenia.

In Switzerland, community psychiatry only exists in specific areas and is insufficiently funded to serve a large number of patients. If psychiatric ambulatory care were developed during recent decades, it took the shape of private psychiatrists and ambulatory clinics, which do not systematically address the needs of people with severe mental disorders when living in the community. Models of care such as "early intervention for psychosis" [13], "assertive community treatment" [14] [15] or "housing first" [16] represent valid alternatives to hospitalizations. If well-provided, these interventions could reduce resource consumption by very heavy users. This local context brings up questions about a real and pragmatic implementation of the conceptual shift towards community in the Swiss psychiatric services. Identification of hospital use patterns may offer a scientific and empirical basis to target more precisely the type and amount of interventions needed for each group of patients. Our results do not allow us to determine if numerous unique episode patients may completely avoid hospitalization at all, and whether alternatives may be found in usual psychiatric or communityoriented primary care. However we can hypothesize that assertive community treatment team may address, more systematically, the needs of revolving door patients, who seem to have alternatives to hospitalization, but not enough support to remain stable in the community. Long stay and permanent stays patients may benefit from supportive housing alternatives such as housing first. However, in our data, these heavy users are few in numbers, with a couple of 
permanent stays and less than fifty long stays or revolving door patients during fifteen months in a catchment area of 620 '000 inhabitants.

When discussing the very heavy users, it is also crucial to remind that psychiatric problems are not the only reasons for inpatient stay. Indeed, using weekly standardized assessment of reasons of hospitalization, it was estimated that social problems could account for up to $20 \%$ of days of hospitalizations in the Department of Psychiatry of Lausanne University Hospital between 2014 and 2016 [17]. Social adaptation difficulties are an integral part of the pathology of some patients. Anxiety, paranoid ideation, cognitive disorders, addictions, behavioral disorders and anti-social traits likely contribute to housing difficulties. Shelter structures are not always able to cope with these difficulties and have a limited tolerance for substance abuse. At a more general level, efforts are still needed to move towards a society that better tolerates and integrates the homeless, patients dropping out of care or refusing care and others living at the margins of society.

\subsection{Conclusion}

This study highlighted that a small minority of patients (4.9\%) occupy about a third of hospital beds and most patients have a single or low number of brief hospital admissions over the three years of the study. Examining the temporal dynamics of hospitalizations with an empirical basis is crucial. These data are generally readily available as statistical tools to describe, visualize and interpret them. Further studies involving multiple centers or a longer time period are warranted to yield samples with greater numbers of heavy users to facilitate between-groups pairwise comparisons. As the present study suggests, heavy users may constitute a very heterogeneous group of individuals with very different clinical presentations, difficulties and needs profiles. 


\section{REFERENCES}

1. Zusman J, Friedman RM, Levin BL. Moving treatment into the community: Implications for psychiatry. Psychiatric Quarterly. 1988;59(2):140-9.

2. Cohen S. Visions of social control: Crime, punishment and classification: Polity Press Cambridge; 1985.

3. McDonnell E, Bartholomew T. Community Treatment Orders in Victoria: Emergent Issues and Anomalies. Psychiatry, Psychology and Law. 1997;4(1):25-36.

4. Lamb HR, Bachrach LL. Some perspectives on deinstitutionalization. Psychiatr Serv. 2001;52(8):1039-45.

5. H+. Les Grandes tendances de la psychiatrie 2017. Available from: http://www.hplus.ch/fr/chiffres faits/moniteur des hopitaux et cliniques de h/psychiatrie/.

6. Gabadinho A, Ritschard G, Mueller NS, Studer M. Analyzing and visualizing state sequences in $R$ with TraMineR. Journal of Statistical Software. 2011;40(4):1-37.

7. Gabadinho A, Ritschard G, Studer M, Müller NS. Mining sequence data in R with the TraMineR package: A users guide for version 1.2. Geneva: University of Geneva. 2009.

8. Gabadinho A, Ritschard G, Studer M, Müller NS, editors. Extracting and rendering representative sequences. International Joint Conference on Knowledge Discovery, Knowledge Engineering, and Knowledge Management; 2009: Springer.

9. Lewis T, Joyce PR. The new revolving-door patients: results from a national cohort of first admissions. Acta Psychiatrica Scandinavica. 1990;82(2):130-5.

10. Haywood TW, Kravitz HM, Grossman LS, Cavanaugh Jr JL. Predicting the" revolving door" phenomenon among patients with schizophrenic, schizoaffective, and affective disorders. The American journal of psychiatry. 1995;152(6):856.

11. Hiday VA, Scheid-Cook TL. Outpatient commitment for" revolving door" patients: compliance and treatment. Journal of Nervous and Mental Disease. 1991.

12. Jeppesen $\mathrm{R}$, Christensen $\mathrm{T}$. Changes in utilization of psychiatric hospital facilities in Denmark by patients diagnosed with Schizophrenia from 1970 through 2012: The advent of 'revolving door'patients. European Psychiatry. 2017;41:S159-S60.

13. Randall JR, Vokey S, Loewen H, Martens PJ, Brownell M, Katz A, et al. A Systematic Review of the Effect of Early Interventions for Psychosis on the Usage of Inpatient Services. Schizophrenia Bulletin. 2015;41(6):1379-86.

14. Clausen H, Ruud T, Odden S, Šaltytė Benth J, Heiervang KS, Stuen HK, et al. Hospitalisation of severely mentally ill patients with and without problematic substance use before and during Assertive Community Treatment: an observational cohort study. BMC Psychiatry. 2016;16:125.

15. Clausen H, Landheim A, Odden S, Šaltytè Benth J, Heiervang KS, Stuen HK, et al. Hospitalization of high and low inpatient service users before and after enrollment into Assertive Community Treatment teams: a naturalistic observational study. International Journal of Mental Health Systems. 2016;10(1):14.

16. Leyla G, Ana S, Marybeth S, Sam T, N. FS. Housing, hospitalization, and cost outcomes for homeless individuals with psychiatric disabilities participating in continuum of care and housing first programmes. Journal of Community \& Applied Social Psychology. 2003;13(2):17186.

17. Golay P, Conus P, Bonsack C. Variabilité des trajectoires d'hospitalisation en psychiatrie : analyses de séquences discrètes. [Variability of hospitalization trajectories in psychiatry: discrete sequence analysis]. Gèmes Journées MODEVAIIA (MODElisation de la VAriabilité Inter et IntrAindividuelle); Marcevol; 2017. 


\section{TABLES}

Table 1. Patients' characteristics in the different clusters.

\begin{tabular}{|c|c|c|c|c|c|c|c|}
\hline & $\begin{array}{l}\text { A - Unique } \\
\text { episode } \\
(n=625)\end{array}$ & $\begin{array}{c}\quad B- \\
\text { Repeated } \\
\text { episodes } \\
\text { (n=223) }\end{array}$ & $\begin{array}{c}A+B- \\
\text { Regular users } \\
(n=848)\end{array}$ & $\begin{array}{c}C-\text { Long stays } \\
(n=15)\end{array}$ & $\begin{array}{l}D \text { - Revolving } \\
\text { door patients } \\
\quad(n=26)\end{array}$ & $\begin{array}{c}E \text { - Permanent } \\
\text { stays } \\
(n=3)\end{array}$ & $\begin{array}{c}C+D+E- \\
\text { Atypical users } n \\
\quad=44)\end{array}$ \\
\hline Age, Mean (SD) & $\begin{array}{c}41.32 \\
(14.11)\end{array}$ & $\begin{array}{c}42.89 \\
(13.48)\end{array}$ & $41.73(13.96)$ & $48.34(16.81)$ & $43.90(9.87)$ & $38.58(12.44)$ & 45.01 (12.85) \\
\hline Gender, Male \% $(\mathrm{N})$ & 47.7 (297) & $50.2(112)$ & $48.3(409)$ & $33.3(5)$ & $32.0(8)$ & $66.7(2)$ & $34.9(15)$ \\
\hline $\begin{array}{l}\text { Presence of } \\
\text { substance use } \\
\text { disorder, \% }(\mathrm{N})\end{array}$ & $35.8(224)$ & $33.2(74)^{\prime}$ & $35.1(298)$ & $20.0(3)$ & $26.9(7)$ & $0.0(0)$ & 22.7 (10) \\
\hline $\begin{array}{l}\text { Presence of } \\
\text { personality disorder, } \\
\%(\mathrm{~N}) \\
\text { Main Diagnostic, \% }\end{array}$ & $29.4(184)$ & $27.8(62)$ & $29.0(246)$ & $33.3(5)$ & $34.6(9)$ & $33.3(1)$ & $34.1(15)$ \\
\hline$(\mathrm{N})$ & $3.3(20)$ & $3.2(7)$ & $3.2(27)$ & $6.7(1)$ & $0.0(0)$ & $0.0(0)$ & $2.3(1)$ \\
\hline Dementia & $11.7(72)$ & $12.2(27)$ & $11.8(99)$ & $20.0(3)$ & $0.0(0)$ & $0.0(0)$ & $7.0(3)$ \\
\hline Alcohol use & $5.7(35)$ & $5.0(11)$ & $5.5(46)$ & $0.0(0)$ & $0.0(0)$ & $0.0(0)$ & $0.0(0)$ \\
\hline Drug use & $11.6(71)$ & $28.4(63)$ & $16.0(134)$ & $40.0(6)$ & $44.0(11)$ & $100.0(3)$ & $46.5(20)$ \\
\hline Schizophrenia & $5.4(33)$ & $5.4(12)$ & $5.4(45)$ & $0.0(0)$ & $4.0(1)$ & $0.0(0)$ & $2.3(1)$ \\
\hline Mania & 36.3 (223) & $35.1(78)$ & $36.0(301)$ & $26.7(4)$ & $40.0(11)$ & $0.0(0)$ & $32.6(14)$ \\
\hline $\begin{array}{l}\text { Depression } \\
\text { Anxiety and stress }\end{array}$ & $18.1(111)$ & $7.2(16)$ & $15.2(127)$ & $6.7(1)$ & $8.0(2)$ & $0.0(0)$ & $7.0(3)$ \\
\hline $\begin{array}{l}\text { related disorder } \\
\text { Behavioral } \\
\text { syndromes } \\
\text { associated with } \\
\text { physiological }\end{array}$ & $1.5(9)$ & $0.9(2)$ & $1.3(11)$ & $0.0(0)$ & $0.0(0)$ & $0.0(0)$ & $0.0(0)$ \\
\hline $\begin{array}{l}\text { disturbances and } \\
\text { physical factors } \\
\text { Personality } \\
\text { disorder }\end{array}$ & $6.5(40)$ & $2.7(6)$ & $5.5(46)$ & $0.0(0)$ & $4.0(1)$ & $0.0(0)$ & $2.3(1)$ \\
\hline
\end{tabular}




\section{FIGURES}

Figure 1. Cluster distribution of the three-year hospitalization trajectories

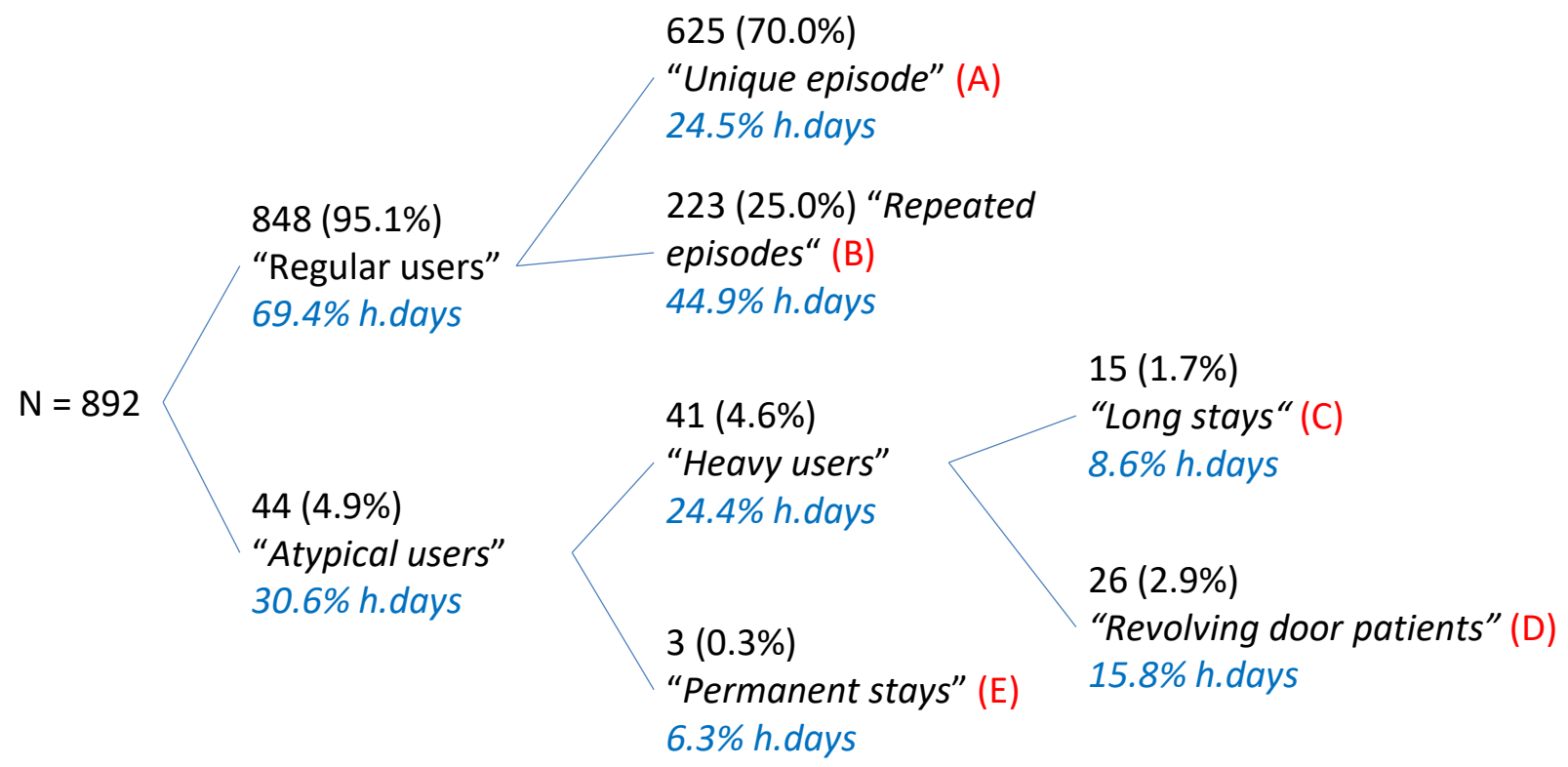

$(\%)=\%$ of patients

$\%$ h.days $=\%$ of hospital days 
Figure 2. Clusters' characteristics

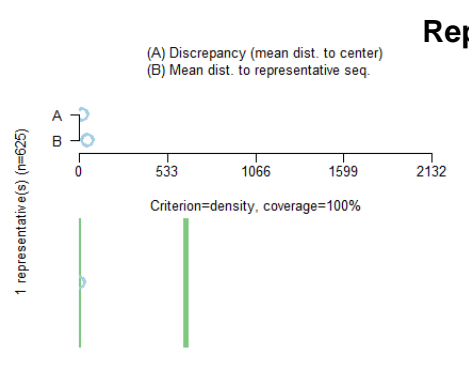

Representatives sequences (left) \& First 10 sequences (right)

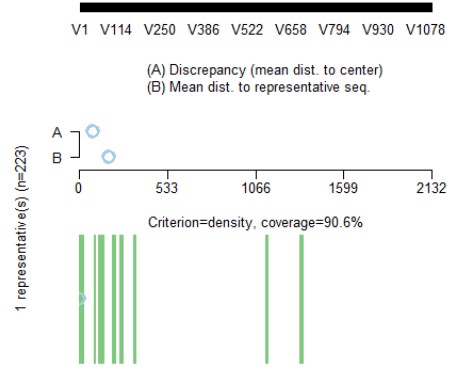

"Unique episode" (A)

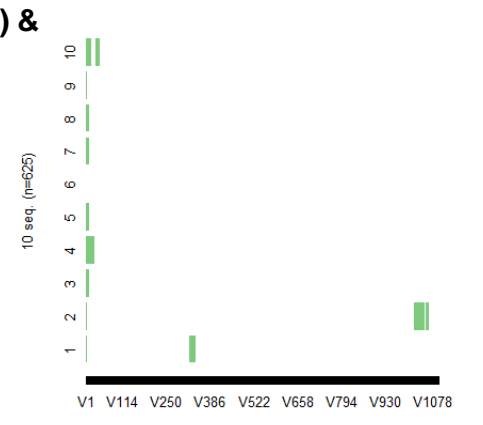

“Repeated episodes” (B)

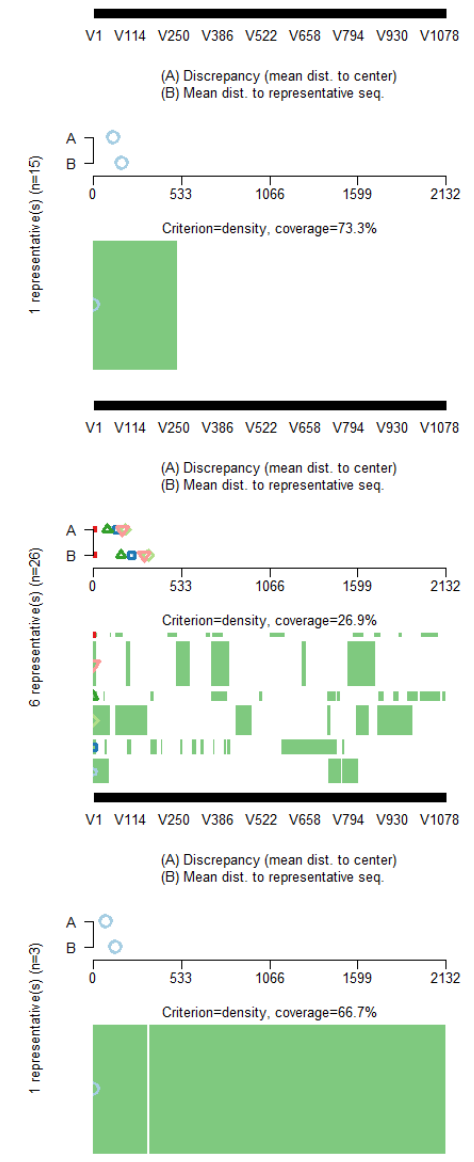
"Repeated episodes" (B)
“Long stays" (C)
"Revolving door patients" (D)
"Permanent stays" (E)
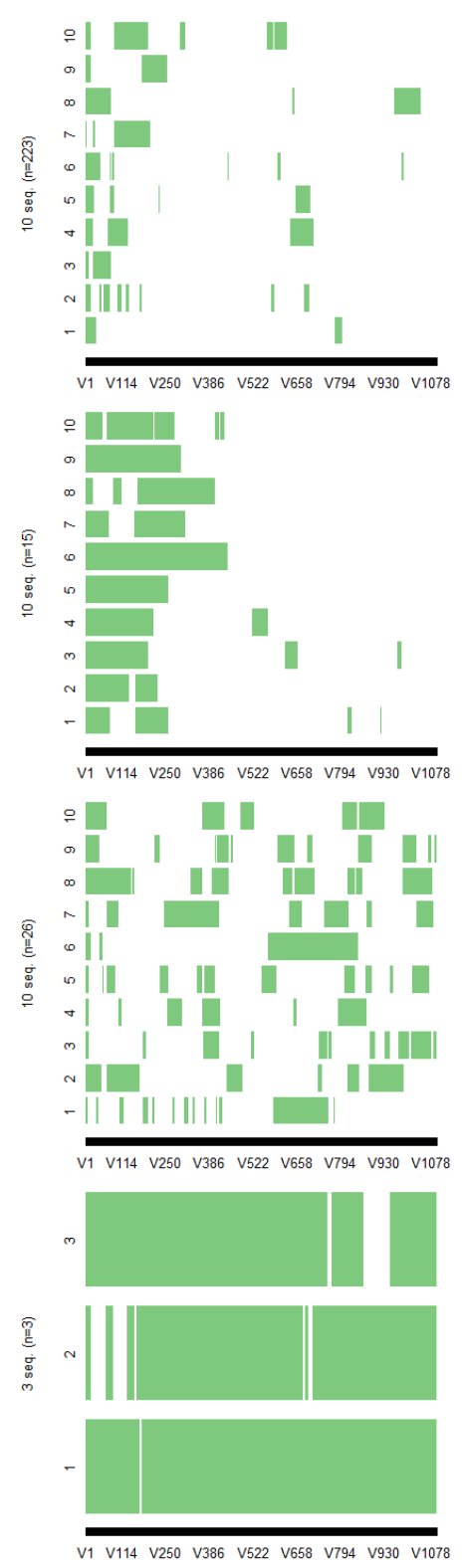
Figure 3. Comparison of total duration of hospitalization between clusters

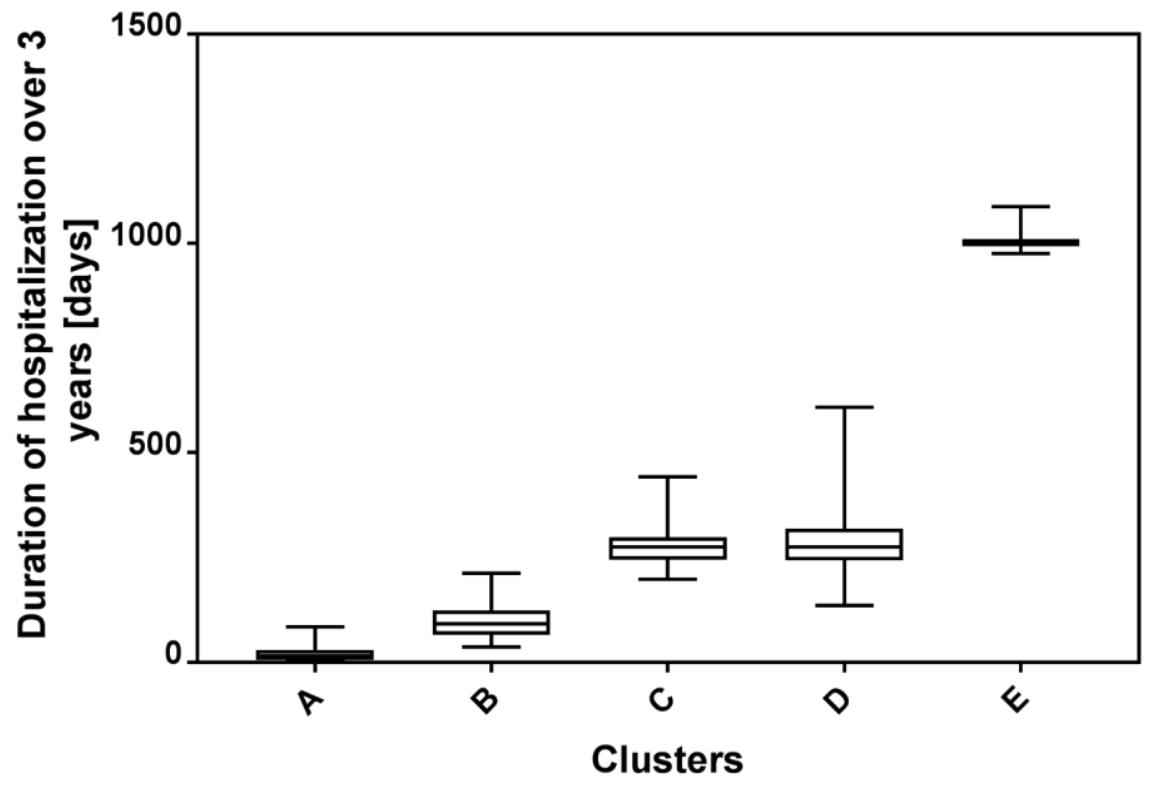

